Ann. Biol. anim. Bioch. Biophys., 1977, 17 (3 A), 309-324.

\title{
Etude quantitative d'organes ou de tissus. III. Méthodes d'estimation du nombre de particules.
}

\author{
par Aline SOLARI
}

Station centrale de Physiologie animale, I.N.R.A.

78350 jouy-en-josas

Summary. Quantitative study of organs and tissues.

III. Methods for estimating particle number.

In this paper the principal methods for estimating the number of particles per unit volume of tissue are presented and discussed. A guide for their use is given. Approximately equal-sized and shaped particles are assumed and only spherical, ellipsoidal and cylindrical shapes give a simple counting procedure. The choice between the different methods depends on the thickness of section and the particle shape. When particle dimension is equal or less than cross-section thickness, choice of method depends on the quality of the resolution power and the form of the particles (table 1). Abercrombie's relationship (1946) is used for a spherical form and if all sections of particles are visible ; in the contrary case, that of Floderus (1944) is employed. If the sphericity concept is not wholly acceptable, Aherne's relationship (1967) may be used.

There are three equal methods from which to choose (De Hoff and Rhines, 1961 ; Weibel and Gomez, 1962 ; Loud ef al., 1965) when particle dimension is greater than crosssection thickness (table 2). The choice is a matter of personal preference, a selection on the basis of easy adaptability. Weibel and Gomez's procedure is the most frequently employed in cellular ultrastructure studies.

These methods are only justified if the concept of random particle distribution is admitted.

\section{Introduction.}

Le nombre $\mathrm{N}$ de cellules ou d'organites cellulaires demeure l'un des critères les plus importants pour caractériser l'activité physiologique d'un tissu ou d'un organe. On détermine $N=V N_{V}$ à partir du volume $V$ du tissu (frais ou fixé) ef du nombre de particules par unité de volume de tissu $N_{V}$, ce dernier paramètre étant obtenu par l'observation quantitative de coupes histologiques. Plusieurs méthodes d'estimation de $\mathrm{N}_{\mathrm{V}}$ ont été mises au point dans les cas où toutes les particules sont de forme identique et principalement sphérique, cylindrique ou ellipsoïdale qui sont les formes géométriques les plus proches de celles de nombreuses cellules ou organites cellulaires. Ces méthodes dépendent de la distribution des tailles des particules et de l'épaisseur de la coupe histologique. Nous présentons dans cet article les différentes relations 
dont nous disposons pour évaluer le nombre de particules par unité de volume ainsi que les conditions d'application pour une meilleure précision.

\section{A. Particules de diamètre inférieur ou équivalent à l'épaisseur de la coupe (coupe épaisse)}

Dans ce qui suit, on suppose les particules réparties au hasard dans le tissu.

Méthode 1.

Considérons une coupe d'épaisseur $t$ sur laquelle on superpose un quadrillage. Dans chaque carré élémentaire, de surface $A$, on dénombre $n$ particules. Si les $n$ particules observées étaient entièrement comprises dans la portion de coupe de volume At, on obtiendrait des estimations correctes du nombre de particules par unité de volume en divisant, pour chaque carré observé, le nombre $n$ par le volume At (Dornfeld, Slater et Scheffe, 1942), soit :

$$
N_{\mathrm{V}}=\mathrm{n} / \text { At } \text {. }
$$

Or, sur les n particules effectivement complées, certaines sont coupées par l'un ou l'autre des deux plans de coupe ; elles n'appartiennent donc pas entièrement au volume-test Af et la relation précédente, bien qu'encore utilisée, fournit dans la plupart des cas une surestimation du nombre de particules par unité de volume. Afin de tenir compte des particules tronquées, on a mis au point des facteurs de correction en faisant l'hypothèse que toutes les particules sont de même taille et de forme sphérique. Ces facteurs sont des estimations de la probabilité qu'une particule de diamètre $D$, coupée

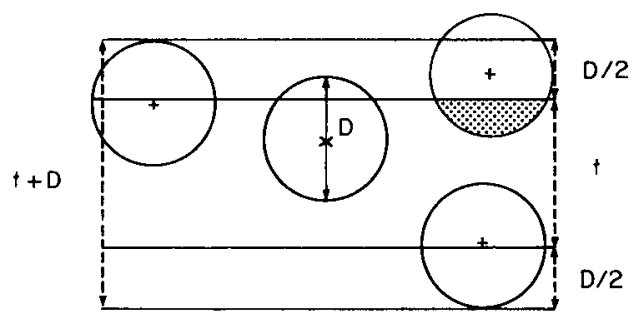

FIG. 1. - Position du centre nucléaire des noyaux vus sur coupe.

section de particules dont le centre n'appartient pas à la coupe.

au hasard par une coupe d'épaisseur $t$, ait son centre dans cette coupe * (fig. 1). Nous retiendrons les plus couramment utilisés, à savoir :

et $\quad t /(t+D-2 k) \quad$ (Abercrombie, 194

* Une section de particule, observée sur coupe, peut avoir son centre dans un volume d'épaisseur $(t+D)$. 
où $k$ est fonction de la plus petite longueur discernable, valeur qui dépend du pouvoir de résolution du microscope : les sections de particules donf l'épaisseur est inférieure à $\mathrm{k}$ ne sont pas discernables. Le nombre de particules dont le centre se trouve dans la coupe est donc relié au nombre total de particules examinées sur la surface-test par les relations suivantes:

$$
N=n t /(t+D) \quad \text { ou } \quad N=n t /(t+D-2 k)
$$

ce qui donne pour le nombre $N_{V}$ de particules par unité de volume

$$
\begin{aligned}
& N_{\mathrm{V}}=N_{\mathrm{A}} /(t+D) \\
& N_{\mathrm{V}}=N_{\mathrm{A}} /(t+D-2 k)
\end{aligned}
$$

où $N_{A}$ représente le nombre de particules entières ou fragmentaires observées par unité de surface. Pour appliquer la relation (2a) d'Abercrombie, on doit pouvoir dénombrer toutes les particules présentes dans la coupe, même celles de taille infiniment petite. Lorsque les hypothèses de sphéricité et d'égalité des tailles sont acceptables, l'épaisseur de la coupe et les diamètres devraient, en foute rigueur, être mesurés perpendiculairement au plan de coupe. Or, les mesures de longueur dans le sens de l'axe optique sont les plus délicates et entachées des plus fortes erreurs. En pratique, on mesure la taille des particules dans le plan horizontal soit par la moyenne des diamètres maximum examinés, soit par leur diamètre moyen apparent multiplié par $(4 / \pi)$ * (fig. 2). Il existe d'autres méthodes d'estimation des diamètres qui seront

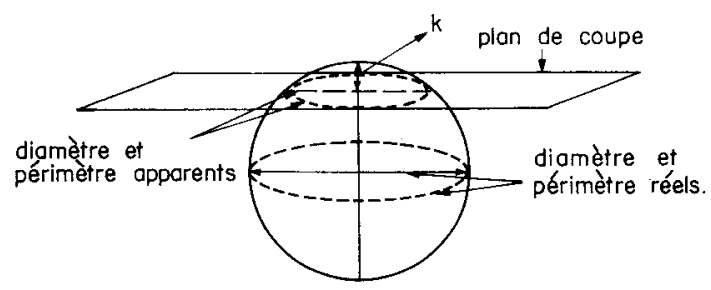

FIG. 2. - Différence entre diamètre réel et apporent.

exposées ultérieurement. La connaissance du diamètre réel $D$ entraîne celle de $k / r$ $(r=$ rayon) qui est fonction du rapport $x$ entre le plus petif rayon mesuré et le plus grand (Freedman, 1974) :

$$
k / r=1-\sqrt{1-x^{2}}
$$

On obtient une bonne précision si l'on mesure un grand nombre de sections de particules.

* Résulfat des probabilités géométriques : la longueur moyenne d'une corde prise au hasard parmi les cordes d'un cercle est égale au diamètre multiplié par $\pi / 4$. 


\section{Méthode 2.}

L'estimation du nombre $N_{v}$ de particules par volume unitaire de tissu a également été abordée par Aherne (1967) qui exprime la relation (2a) en fonction du périmètre équatorial des particules $\mathrm{Pe}$, soit

$$
N_{V}=N_{A} /(t+P e / \pi)
$$

Pour éviter l'évaluation directe du périmètre équatorial, l'auteur utilise les résultats relatifs à l'estimation des longueurs de courbe située dans un plan (Solari, 1975). On superpose à la coupe histologique un système de parallèles équidistantes (intervalle $=\mathrm{d}$ ) et on observe le nombre d'intersections des limites des particules et des parallèles (fig. 3). La quantité $I d \pi / 2(1=$ nombre moyen d'intersections par sec-

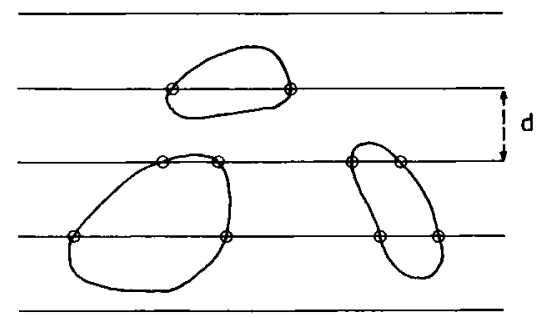

FIG. 3. - Estimation des longueurs por la méthode des intersections.

0 : points d'intersections des limites des particules et des parallèles du réseau.

tion de particules) fournit une estimation du périmètre * moyen $\bar{p}$. Quand tous les organites sont des sphères de même rayon $r$, Aherne note que le périmètre moyen permet d'obtenir une valeur approchée du périmètre équatorial puisque :

$$
1 \leqslant \mathrm{Pe} / \overline{\mathrm{P}} \leqslant(4 / \pi)
$$

Le problème consiste donc à trouver le meilleur facteur de proportionnalité entre le périmètre équatorial $p_{e}$ ef le périmètre moyen $\bar{p}$ : valeur comprise entre 1 ef $4 / \pi$. Puisque $\bar{p}=I d \pi / 2$, si on pose $p_{e}=j d \pi / 2$ pour $I \leqslant j \leqslant(4 / \pi)$ I, la relation (4) devient :

$$
N_{V}=N_{A} /(t+j d / 2)
$$

dite approximation de Aherne car pratiquement on utilise $\mathbf{j}=1$.

En effet, lorsque la taille des particules est inférieure ou équivalente à l'épaisseur de la coupe, le périmètre équatorial se confond avec le périmètre moyen et on prendra $\mathbf{j}=1$. Au contraire, lorsque la taille est largement supérieure à l'épaisseur

$$
\mathbf{j}=\mathrm{I}(4 / \pi)=1,27 \mathrm{I} \text {. }
$$

* Pour déterminer une mesure réelle $L$ on utilise l'équation suivante $L=L_{M}(1000 / G)$ où $L_{M}$ représente la longueur en $\mathrm{mm}$ après un grossissement $G$ et $L$ la longueur en microns lorsque $L_{M}$ est ramenée à l'échelle du tissu. 


\section{Méthode 3.}

D'autres expérimentateurs (Ebbesson et Tang, 1965) proposent de dénombrer les particules dans des coupes adjacentes d'épaisseurs différentes, ce qui évite la mesure des diamètres ou périmètres. lls montrent que :

$$
N_{1}=\left(n_{1}-n_{2}\right) t_{1} /\left(t_{1}-t_{2}\right) \quad \text { et } \quad N_{2}=\left(n_{1}-n_{2}\right) t_{2} /\left(t_{1}-t_{2}\right)
$$

où $N_{1}$ et $N_{2}$ sont les nombres de particules dont le centre se trouve dans les coupes d'épaisseur $t_{1}$ et $t_{2}\left(t_{1}>t_{2}\right)$ et $n_{1}, n_{2}$ les nombres effectivement observés. Cette méthode serait équivalente à celle de Floderus.

Lorsque les particules sont de taille différente et de forme quelconque, Underwood (cité par Freedman, 1974) propose une variante de la méthode précédente. Celle-ci consiste à rechercher la régression du nombre observé de particules en fonction de l'épaisseur des coupes. Le nombre par unifé de volume correspond au coefficient de régression que l'on calcule par la méthode des moindres carrés.

En conclusion, lorsque la dimension des particules est inférieure ou égale à l'épaisseur de la coupe, le choix de la méthode d'estimation dépend de la qualité du pouvoir

\section{TABLEAU 1}

Estimation du nombre $N_{v}$ de particules par unité de volume lorsque les dimensions des particules sont inférieures ou équivalentes à l'épaisseur de la coupe $(D \leqslant t) *$

\begin{tabular}{|c|c|c|c|c|}
\hline $\begin{array}{l}\text { Qualité du pouvoir } \\
\text { de résolution }\end{array}$ & $\begin{array}{c}\text { Distribution } \\
\text { des tailles } \\
\text { des particules }\end{array}$ & $\begin{array}{l}\text { Forme des } \\
\text { particules }\end{array}$ & Conditions complémentaires & $\begin{array}{c}\text { Formule à employer pour } \\
\text { l'estimation de } N_{v}\end{array}$ \\
\hline \multirow{4}{*}{$\begin{array}{l}\text { Toutes les sections } \\
\text { de particules } \\
\text { sont visibles } \\
(k / r<0,06)\end{array}$} & \multirow{3}{*}{$\begin{array}{l}\text { Egalité } \\
\text { des tailles } \\
\text { ou légère } \\
\text { variabilité }\end{array}$} & \multirow[b]{2}{*}{ Sphérique } & Estimation du diamètre & \multirow{2}{*}{$\frac{N_{A} /(t+I d / 2) \quad \text { Aherne }}{N^{\prime}}$} \\
\hline & & & $\begin{array}{l}\text { Diamètre inférieur ou équiva- } \\
\text { lent à l'épaisseur de coupe }\end{array}$ & \\
\hline & & $\begin{array}{c}\text { Non } \\
\text { sphérique }\end{array}$ & $\begin{array}{l}\text { Dimension moyenne observée } \\
<0,8\end{array}$ & e $\mathrm{N}_{\mathrm{A}} /(\mathrm{t}+\mathrm{Id} / 2) \quad$ Aherne \\
\hline & $\begin{array}{c}\text { Grande } \\
\text { variabilité }\end{array}$ & $\begin{array}{c}\text { Sphérique } \\
\text { ou } \\
\text { quelconque }\end{array}$ & $\begin{array}{l}\text { Etude de la distribution des } \\
\text { tailles }\end{array}$ & $\begin{array}{l}\text { Calcul de } N \text { pour chaque } \\
\text { classe de taille** }\end{array}$ \\
\hline \multirow{3}{*}{$\begin{array}{l}\text { Certaines sections } \\
\text { de particules sont } \\
\text { indiscernables }\end{array}$} & \multirow{3}{*}{$\begin{array}{l}\text { Egalité } \\
\text { des tailles } \\
\text { ou légère } \\
\text { variabilité }\end{array}$} & Sphérique & Estimation de $D$ ef $k$ & $\mathrm{~N}_{\mathrm{A}} /(\mathrm{t}+\mathrm{D}-2 \mathrm{k})$ \\
\hline & & \multirow{2}{*}{$\begin{array}{c}\text { Non } \\
\text { sphérique }\end{array}$} & $\begin{array}{l}\text { Dimension moyenne observée } \\
<2+\end{array}$ & $\mathrm{N}_{\mathrm{A}} /(\mathrm{t}+\mathrm{Id} / 2) \quad$ Aherne \\
\hline & & & $\begin{array}{l}\text { Dimension moyenne observée } \\
<0,04\end{array}$ & $N_{A} / t$ \\
\hline$(0,06 \leqslant k / r \leqslant 0,15)$ & $\begin{array}{c}\text { Grande } \\
\text { variabilité }\end{array}$ & $\begin{array}{c}\text { Sphérique } \\
\text { ou } \\
\text { quelconque }\end{array}$ & $\begin{array}{l}\text { Etude de la distribution des } \\
\text { tailles }\end{array}$ & $\begin{array}{l}\text { Calcul de } N \text { pour chaque } \\
\text { classe de taille ** }\end{array}$ \\
\hline
\end{tabular}

* $t=$ épaisseur de la coupe $; D=2 r=$ diamètre des particules ; $k=$ épaisseur de la plus petite particule discernable; $N_{A}=$ nombre observé de sections de particules par unité de surface $I=$ nombre moyen d'intersections par particule; $d=$ intervalle entre les parallèles d'un réseau.

** Les méthodes seront présentées dans un article ultérieur. 
de résolution du microscope * de la forme des particules et de la distribution de leur taille (Freedman, 1974) (tabl. 1).

Un pouvoir de résolution parfait ou presque ef des particules sphériques de diamètres identiques ou peu différents permettent d'utiliser la relation simple d'Abercrombie. Cependant, l'hypothèse de sphéricité s'avère restrictive car si on peut l'accepter pour les noyaux cellulaires au repos ou les nucléoles, il n'en est pas de même pour les fuseaux mitotiques (Marrable, 1962), ni pour des organites tels que les mitochondries qui s'apparentent, le plus souvent, à des formes cylindriques. On utilise alors de préférence l'approximation de Aherne pour $j=1$ qui donne, selon Freedman, une surestimation du nombre inférieure à 5 p. 100 pourvu que les dimensions moyennes observées soient inférieures à 0,8 fois l'épaisseur de la coupe.

Lorsque le pouvoir de résolution est bon, sans être parfait, la formule d'Abercrombie doit être remplacée par celle de Floderus. On applique celle de Aherne lorsqu'on ne peut accepter sans réserve l'hypothèse de sphéricité des particules.

S'il existe une grande variabilité des tailles, il est nécessaire de faire une étude préalable de leur distribution.

\section{B. Particules de dimensions supérieures à l'épaisseur de la coupe (coupe fine)}

La validité des méthodes présentées dans cette partie suppose que l'on puisse assimiler la coupe histologique à un plan et que les particules soient réparties au hasard dans le tissu.

\section{Méthode 1.}

Afin d'étudier les transformations métallurgiques et d'analyser les relations entre le nombre de microstructures et les propriétés des métaux, De Hoff et Rhines (1961), De Hoff (1968) ont montré que le nombre $N_{V}$ de particules présentes par unité de volume et le nombre $N_{A}$ de sections de particules par unité de surface étaient liés par la relation suivante :

$$
\mathrm{N}_{\mathrm{V}}=\mathrm{N}_{\mathrm{A}} / \overline{\mathrm{D}}_{\mathrm{V}}
$$

où $\bar{D}_{\mathrm{V}}$ représente le diamètre tangent moyen des particules, c'est-à-dire la distance moyenne entre les plans parallèles et tangents aux particules (fig. 4). Elle a été obtenue à partir de la probabilité qu'un plan intercepte une particule. Si la forme est sphérique, la distance $\bar{D}_{V}$ correspond au diamètre, c'est-à-dire en pratique au plus grand diamètre mesuré. On retrouve ainsi la relation d'Abercrombie pour une épaisseur de coupe infiniment petite par rapport au diamètre. Dans ce cas particulier, on obtient

* Noté k/r par analogie aux sphères (Freedman, 1974). 
une approximation de la relation (6) en utilisant la méthode des intersections (fig. 3-5) (Fullman, 1953).

$$
\mathrm{N}_{\mathrm{V}}=\pi \mathrm{N}_{\mathrm{A}}^{2} / 2 \mathrm{I}_{\mathrm{L}}
$$

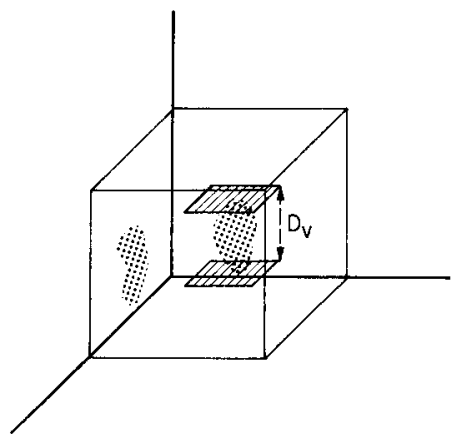

FIG. 4. - Définition de $\vec{D}_{V}$.

OU

$$
N_{V}=\pi N_{\mathbf{A}}^{2} / 4 N_{L}
$$

avec $I_{\mathbf{L}}$ : nombre d'intersections des limites des particules par unité de longueur de ligne échantillon

$N_{L}$ : nombre de sections de sphères par unité de longueur de ligne échantillon (fig. 5).

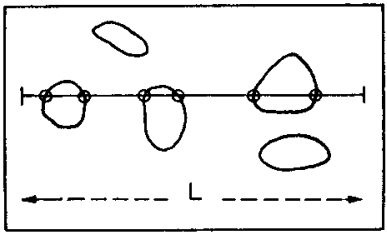

FIG. 5. - Estimation du nombre de particules par la méthode des intersections.

$\mathrm{L}=$ longueur de la droite $=$ longueur unitaire $; l_{\mathrm{L}}=6=$ nombre d'intersections par unité de pongueur de ligne-échantillon; $N_{L}=3=$ nombre de particules coupées par unité de longueur de ligne-échantillon.

\section{Méthode 2.}

Dans la perspective d'une analyse quantitative précise du poumon humain, Weibel et Gomez (1962) ont élaboré une nouvelle relation qui leur permet de déterminer aisément le nombre d'alvéoles et conduits alvéolaires. A partir des principes 
de Delesse et Rosiwal les auteurs ont exprimé le nombre de particules par unité de volume en fonction des coefficients de forme et de distribution des tailles. Lorsque les particules sont de même forme et de même taille, ils obtiennent :

$$
N_{V}=N_{A}^{3 / 2} /\left(\beta \sqrt{\left.V_{V}\right)}\right.
$$

ef lorsqu'elles ne diffèrent que par leur dimension :

$$
N_{V}=\left(K N_{A}^{3 / 2}\right) /\left(\beta \sqrt{V_{V}}\right)
$$

où $V_{V}$ représente la part de volume occupé par les particules dans le tissu. Elle est estimée par la méthode des numérations ponctuelles (Solari, 1973) qui consiste à observer la proportion de points qui se superpose aux sections de particules (fig. 6) $\left(V_{V}=P_{T} / P\right)$,

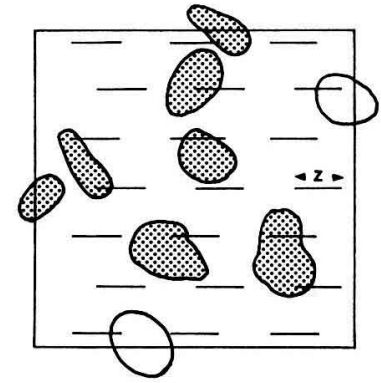

(a)

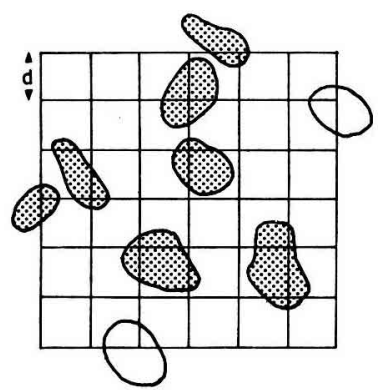

(b)

FIG. 6. - Estimation du nombre de particules : emploi de divers réseaux :

a) $P=42=$ nombre total de points; $P_{T}=7=$ nombre de points sur les particules; $L_{T}=21 \mathrm{Z}$ ( $Z=$ longueur des segments de droites); $N_{A}=7=$ nombre de sections de particules dans la surface $A ; l=9=$ nombre d'intersections.

b) $P=36=$ nombre total d'intersections = nombre de carrés de côté $d ; P_{T}=8=$ nombre de points sur les particules; $L_{T}=2 \mathrm{dP} ; I=16=$ nombre d'intersections ; $N_{A}=7=$ nombre de sections de particules dans la surface $A$.

$\beta$ : coefficient sans dimension ; c'est une caractéristique géométrique des particules qui reflète à la fois leur forme et leurs dimensions*. On l'obtient graphiquement (fig. $7 b$ ) en fonction de $\varepsilon$ rapport axial des particules, c'est-à-dire : diamètre sur longueur dans le cas de cylindre circulaire droit ou de l'axe de rotation sur l'axe perpendiculaire pour les particules ellipsoïdales. On note que pour :

$\varepsilon>1 \beta$ varie très légèrement quand $\varepsilon$ augmente et ceci quelle que soit la forme ;

$* \beta=\mathbf{v} / \overline{\mathbf{a}}^{3 / 2}$ où

$\checkmark=$ volume d'une particule ;

$\bar{a}=$ aire moyenne de toutes les sections de particule réalisées par un plan placé dans toutes les orientations possibles (fig. 7a). 
$\varepsilon<1$ la variation de $\beta$ est importante et peut même atteindre des valeurs élevées quand $\varepsilon$ diminue. Pour $\varepsilon=0,05, \beta$ atteint la valeur 6,155 lorsque les particules sont cylindriques.

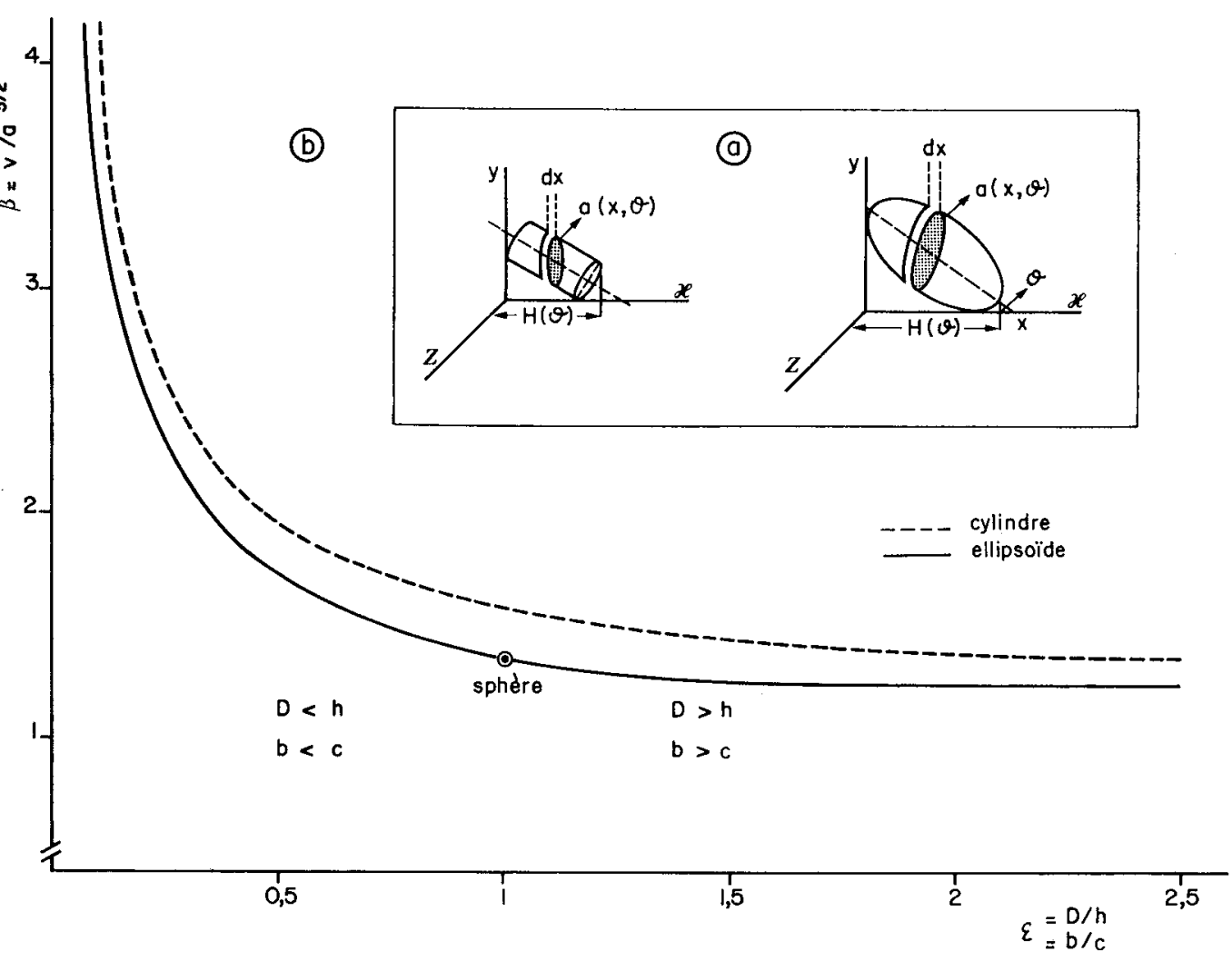

FIG. 7

a) Estimation de $a=\frac{2}{\pi} \int_{0}^{\pi / 2} a(\theta) d \theta$ avec $a(\theta)=v / H(\theta) ; H(\theta)=$ longueur de la projection de la particule sur l'axe $x$.

b) Valeur du coefficient $\beta$ en fonction de $\varepsilon$ pour les particules de forme cylindrique ( $D=$ diamètre, $h=$ hauteur) et ellipsoïdale $(c=a x e$ de rotation, $b=$ axe perpendiculaire). ( $D$ 'après Weibel et Gonez, 1962).

Le coefficient $\beta$ est indépendant du rapport axial pour les sphères et les demisphères ; les valeurs sont respectivement égales à $1,382=\sqrt{6 / \pi}$ et 1,360 .

$K$ : coefficient légèrement supérieur à 1 qui dépend de la distribution des tailles. Pour une distribution normale, avec un coefficient de variation égal à 25 p. 100 , $K$ est peu différent de 1,07 (Weibel, Kistler ef Scherle, 1966). En général, dans les éfudes biologiques, la valeur de $\mathrm{K}$ se trouve comprise entre 1,01 ef 1,10 (Weibel, 1969).

$N_{A}$ : nombre observé de sections de particules par unité de surface. On dénombre 
sur coupes histologiques toutes les sections d'organiles entièrement comprises dans l'aire d'observation et celles interceptées par les bords gauche ef supérieur, mais on ne comptera pas celles coupées par les bords droit et inférieur (Nourtier, 1971).

Les deux relations précédentes ont été vérifiées expérimentalement, par les auteurs, en incluant dans un volume donné de gélatine un nombre connu de pois et de haricots. Après solidification, le bloc de gélatine a été coupé et on a compté le nombre de sections de pois ou de haricots sur chaque coupe. En assimilant les pois à des ellipsoïdes $(\varepsilon=0,85$ donc $\beta=1,42$ ) et les haricots à des cylindres (avec $\varepsilon$ variable), les auteurs trouvent peu de différence entre les nombres calculés et ceux effectivement inclus dans la gélatine. Les écarts respectifs sont en moyenne de 1,4 p. 100 et 3,3 p. 100 (Weibel et Gomez, 1962).

Lorsque les particules n'ont aucune des formes analysées précédemment, on les assimile à la forme géométrique simple la plus voisine, ce qui n'est pas toujours aisé. C'est le cas, ainsi que le note Aherne (1967), des mitochondries des cellules adipeuses

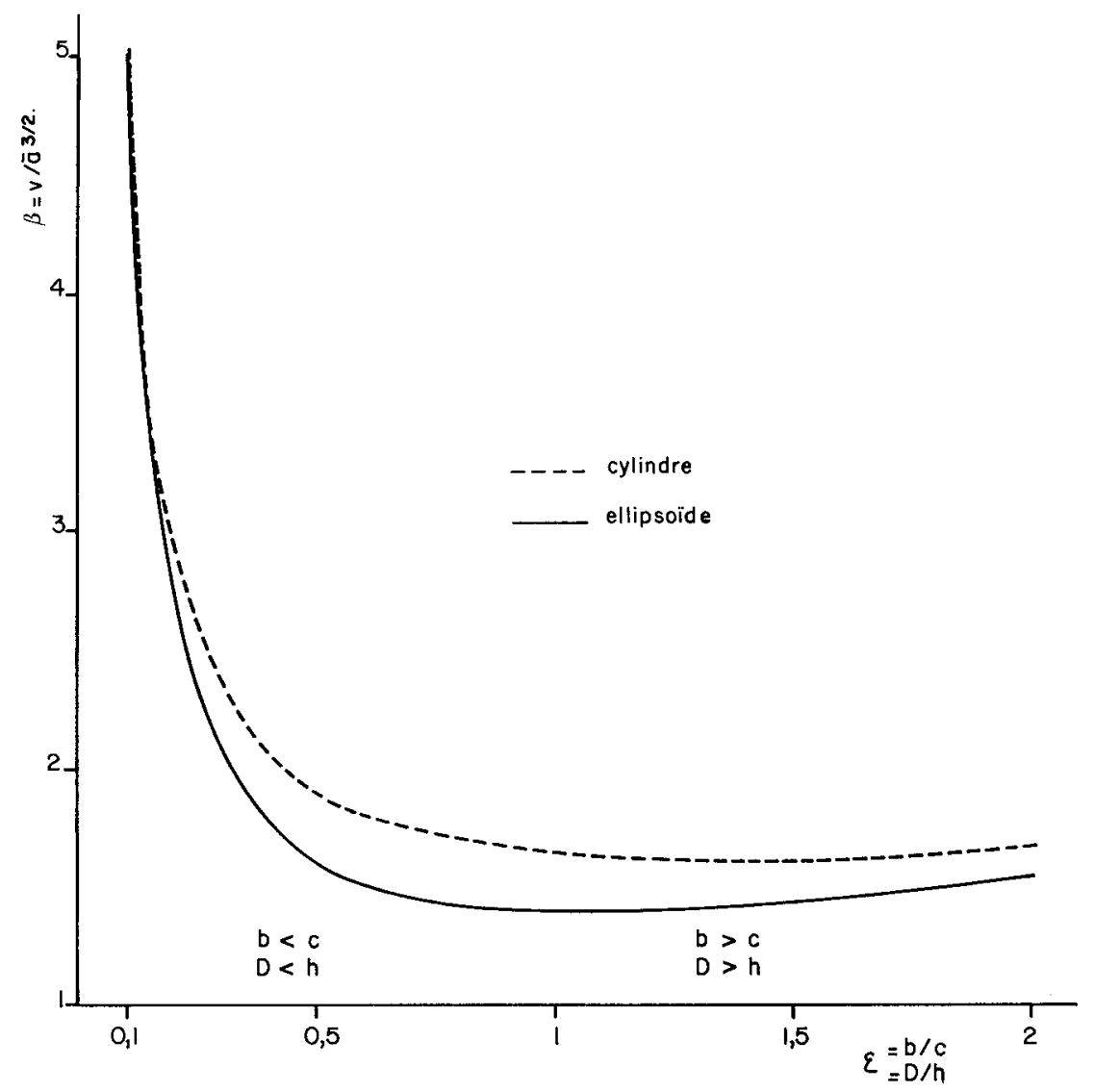

FIG. 8. - Valeur du coefficient $\beta$ en fonction de $\varepsilon$ pour les parficules de forme cylindrique ( $D=$ diamètre, $h=$ hauteur) ef ellipsoïdale $(c=a x e$ de rotation, $b=a x e$ perpendiculaire) (d'après Lindberg ef Worwerk, 1970). 
brunes du lapin nouveau-né, qui sont en fait des cylindres coiffés à chaque extrémité par un hémisphère. On peut alors se demander quelle valeur choisir pour $\beta$ qui devient ambigu.

La détermination de la fonction reliant $\beta$ à $\varepsilon$ suppose les particules réparties uniformément dans le tissu. Pour l'analyse des cellules réparties selon un gradient (ex. : tissu d'origine tumorale), Lindberg et Worwerk (1970) ont recherché une relation plus générale entre $\beta$ et $\varepsilon$ (fig. 8 ). Les valeurs obtenues ne diffèrent pas significativement de celles calculées à partir de la relation mise au point par Weibel et Gomez. Pour l'estimation du nombre de particules, l'imprécision sur $\beta$ a moins d'importance que le biais introduit par un échantillonnage non représentatif du tissu.

\section{Méthode 3.}

Loud (1968), pour les cellules hépatiques, propose d'utiliser la relation simple suivante qui exprime le nombre de particules par unité de volume en fonction de la part de volume $V_{V}$ qu'elles occupent dans le tissu et de leur volume individuel $v$, soit :

$$
N_{\mathrm{V}}=\mathrm{V}_{\mathrm{v}} / \mathrm{v}
$$

Pour appliquer cette méthode il faut également supposer l'homogénéité des formes et l'égalité das tailles.

Pour des particules de forme sphérique, on évalue le volume individuel à partir du rapport surface à volume $S_{V}=s / v$ avec $s=4 \pi r^{2}$ et $v=(4 / 3) \pi r^{3}$ soit $S_{V}=3 / r=$ $2 I_{L}$, ce qui donne la valeur du rayon $r$ et par suite celle du volume*.

La connaissance des dimensions par l'intermédiaire de $S_{V}$ n'est pas toujours aussi aisée. Si nous prenons le cas d'une particule cylindrique de diamètre $D$ et de hauteur $h$, sa surface et son volume sont égaux d̀ $s=\pi D(D+h) / 2$ ef $v=\pi D^{2} h / 4$. Le rapport surface à volume s'écrit :

$$
\mathrm{S}_{\mathrm{v}}=\mathrm{s} / \mathrm{v}=2(\varepsilon+2) / \mathrm{D} \quad \text { avec } \quad \varepsilon=\mathrm{D} / \mathrm{h} .
$$

On en déduit la relation suivante :

$$
v=2 \pi(\varepsilon+2)^{3} / \varepsilon S_{V}^{3}
$$

qui exprime le volume en fonction de $S_{V}$ mais aussi de $\varepsilon$. vient :

En égalant les relations $(9)$ et (11) et en remplaçant $v$ par l'expression (12), il

$$
N_{A}^{3 / 2} /\left(\beta V_{V}^{1 / 2}\right)=V_{V} \varepsilon S_{V}^{3} / 2 \pi(\varepsilon+2)^{3}
$$

Si on pose :

$$
2 \pi(\varepsilon+2)^{3} / \beta \varepsilon=F(\varepsilon)
$$

* Aherne (1967) utilise le volume $v$ et la surface s de la particule dans son approche d'estimation de $N_{v}$ qui est présenté comme une fonction de $k=v^{2 / 3} / s$. Cette méthode ne sera pas détaillée car le coefficient sans dimension $k$ dépend de la forme et des dimensions des particules et n'offre donc pas sur le plan pratique de différence avec celle proposée par Weibel et Gomez (1962). 
on obtient en rassemblant dans un même membre les caractéristiques mesurables

$$
F(\varepsilon)=V_{V}^{3 / 2} S_{V}^{3} / N_{A}^{3 / 2}
$$

On mesure le membre de droiłe de l'équation (13) par les méthodes des numérations ponctuelles et des intersections. La valeur obtenue permet de déterminer le rapport axial (fig. 9, courbe 1) * et par voie de conséquence le volume ou le coeffi-

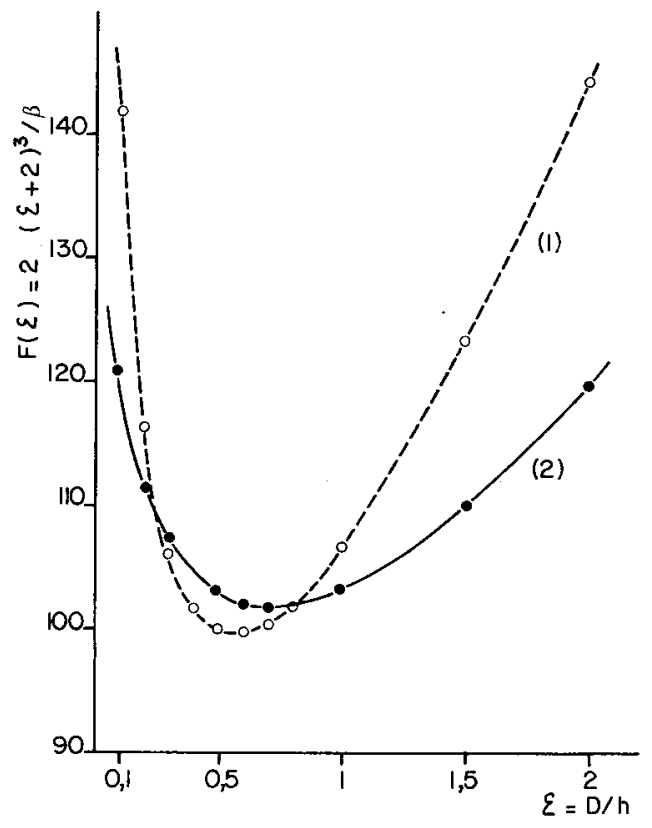

FIG. 9. - Valeur de $F(\varepsilon)$ en fonction de $\varepsilon$ pour les particules de forme cylindrique.

O.... O courbe 1 (Loud ef al., 1965)

courbe 2 (Mathieu ef Messier, 1973).

cient $\beta$ qui permettent à leur tour d'estimer $N_{V}$ (Loud, Barany et Pack, 1965 ; Nourtier, 1971). La courbe 1, calculée avec le $\beta$ de Weibel et Gomez (fig. 7), donne deux valeurs possibles pour $\varepsilon$ de part et d'autre de 0,6 dont une seule convient. En utilisant le $\beta$ de Lindberg (fig. 8), on obtient une fonction qui décrit un minimum pour $\varepsilon \# 0,73$ (fig. 9 , courbe 2) (Mathieu et Messier, 1973). A une même valeur observée de $F(\varepsilon)$ correspondent également deux valeurs de $\varepsilon$, ceci entraîne une imprécision lorsque le rapport axial est peu différent de 1 . Pour $\varepsilon$ compris entre 0,1 et 1,5 , la différence entre les valeurs des courbes 1 et 2 est toujours inférieure à 15 p. 100 .

En résumé (tabl. 2), la méthode de De Hoff et Rhines (méthode 1) est d'utilisation

* On établit la figure 9 en calculant $F(\varepsilon)$ pour différentes valeurs de $\varepsilon$ puisque pour chacune d'elles on connaît le $\beta$ correspondant (fig. 7 ou 8). 
difficile lorsque les particules ne sont pas de forme sphérique. Il convient donc, avant tout dénombrement, d'étudier leur forme et la distribution des tailles afin de calculer leur diamètre : ce qui nécessite beaucoup de temps. Cette méthode a été appliquée en particulier dans les travaux de Nussdorfer et coll. (1974) qui ont analysé les effets de ACTH sur les composants cellulaires de la zona fasciculata du cortex surrénalien de rat.

TABLEAU 2

Estimation du nombre $N_{V}$ de particules par unité de volume lorsque les dimensions des particules sonf supérieures d̀ l'épaisseur de la coupe $(D>t) *$

\begin{tabular}{|c|c|c|c|c|}
\hline $\begin{array}{l}\text { Forme des } \\
\text { particules }\end{array}$ & $\begin{array}{l}\text { Distribution } \\
\text { des tailles }\end{array}$ & $\begin{array}{l}\text { Formules à } \\
\text { I'estima }\end{array}$ & $\begin{array}{l}\text { employer pour } \\
\text { tion de } N_{v}\end{array}$ & $\begin{array}{l}\text { Evaluation des paramètres } \\
\text { de forme }\end{array}$ \\
\hline \multirow{4}{*}{ Sphérique } & \multirow{3}{*}{$\begin{array}{l}\text { Égalité } \\
\text { des tailles } \\
\text { ou légère } \\
\text { variabilité }\end{array}$} & $\begin{array}{l}N_{A} / D \\
\Pi N_{A} / S_{V}\end{array}$ & $\begin{array}{l}\text { De Hoff, Rhines } \\
\text { Fullman }\end{array}$ & \\
\hline & & $\mathrm{N}_{\mathrm{A}}^{3 / 2} /\left(\beta \sqrt{\left.\bar{V}_{\mathrm{v}}\right)}\right.$ & Weibel, Gomez & $\beta=1,382$ \\
\hline & & $V_{v} / v$ & Loud & $v=(4 / 3) \Pi r^{3}$ où $r=3 / 2 I_{L}$ \\
\hline & $\begin{array}{c}\text { Grande } \\
\text { variabilité }\end{array}$ & $\begin{array}{l}\text { Calcul de } N_{v} \text { por } \\
\text { taille }\end{array}$ & ur chaque classe de & \\
\hline \multirow{2}{*}{$\begin{array}{c}\text { Ellipsoïdale } \\
\text { ou } \\
\text { cylindrique }\end{array}$} & $\begin{array}{l}\text { Egalité } \\
\text { des tailles }\end{array}$ & $N_{A}^{3 / 2} /\left(\beta \sqrt{V_{v}}\right)$ & Weibel, Gomez & $\beta$ fonction de $\varepsilon$ Fig. 7 \\
\hline & $\begin{array}{l}\text { Variabilité } \\
\text { des tailles }\end{array}$ & \multicolumn{2}{|c|}{$\left(\mathrm{KN}_{\mathrm{A}}^{3 / 2}\right) /\left(\beta \sqrt{\mathrm{V}_{\mathrm{V}}}\right)$ Weibel } & $\beta$ fonction de $\varepsilon$ Fig. $7 \quad K \not 1,07$ \\
\hline Cylindrique & Egalité & $v_{v} / v$ & Loud & $\begin{array}{l}\varepsilon \text { à partir de Fig. } 9_{-3}^{1} \\
v=2 \Pi(\varepsilon+2)^{3} / \varepsilon s_{v}\end{array}$ \\
\hline $\begin{array}{c}\text { Quelconque } \\
\text { ou } \\
\text { de préférence } \\
\text { sphérique }\end{array}$ & $\begin{array}{c}\text { Egalité } \\
\text { des tailles } \\
\text { ou légère } \\
\text { variabilité }\end{array}$ & $N_{A} /(t+1,27 \mid d)$ & Aherne & \\
\hline
\end{tabular}

* $D=2 r=$ diamètre de la particule $; t=$ épaisseur de la coupe $; N_{A}=$ nombre observé de sections de particules par unité de surface ; $S_{V}=$ densité de surface $=2 I_{L} ; I_{L}=$ nombre d'intersections des limites cellulaires par unité de longueur de ligne-échantillon; $V_{V}=$ volume relatif $=P_{T} / P$.

La méthode de Weibel et Gomez (méthode 2) est de portée plus générale puisqu'on peut déterminer également, par son intermédiaire, le nombre d'organites de forme approximativement ellipsoïdale ou cylindrique et de taille hétérogène. Sa précision dépend de celle de $\beta$ et donc de la connaissance des formes et des dimensions. Ces critères connus, elle trouve sa pleine justification dans les travaux de routine pour sa plus grande rapidité d'exécution. Cette méthode est fréquemment utilisée en biologie (Weibel, 1963 ; Dunnill, 1962, 1964 ; Helander et Bloom, 1973 ; Mausle et Scherrer, 1974). Par contre, la formulation simple de la méthode de Loud (méthode 3) est un grand avantage ; on l'emploie d'emblée lorsqu'on ne connaît aucun autre procédé. On peut l'étendre aux dénombrements de particules de forme complexe pourvu que l'on puisse obtenir une estimation correcte de leur volume individuel. La méthode de 
Loud combinée à celle de Weibel a été appliquée à la quantification des mitochondries pendant le cycle cellulaire du hamster (Dewey et Fuhr, 1976).

La comparaison des trois méthodes d'estimation a été réalisée empiriquement. Elles ont été appliquées à la détermination du nombre d'organifes par unité de volume de cytoplasme de macrophage du péritoine de rat (Mayhew, 1972). On constate un accord excellent entre les valeurs obtenues dans l'hypothèse de sphéricité. On trouve par exemple 24,19 granules $/ \mu \mathrm{m}^{3}$ (De Hoff et Rhines), 24,21 granules $/ \mu \mathrm{m}^{3}$ (Weibel et Gomez) et 22,92 granules $/ \mu \mathrm{m}^{3}$ (Loud) chez des rats ayant subi le même traitement. Cependant, la concordance ou la différence des résultats n'est pas un argument suffisant pour accepter ou réfuter les méthodes précédentes. En effet, celles-ci ne sont pas indépendantes puisqu'elles sont toutes fonction des dimensions de la particule. On trouve en effet au dénominateur le diamètre de la particule (méthode 1), son volume (méthode 3) ou le coefficient $\beta$ (méthode 2) qui dépend par définition du volume de la particule. Il s'ensuit que le choix de l'un des trois procédés (ou de la combinaison méthode 2 et 3) demeure une question de préférence personnelle dictée par l'ensemble des mesures stéréologiques qu'il convient de faire et par la forme, simple ou complexe, des particules (Mathieu et Messier, 1973 ; Elias, Hennig et Schwartz, 1971 ; Weibel, 1969). Quant au choix du réseau, on peut utiliser celui qui permet d'évaluer en même temps les différents paramètres stéréologiques $V_{V}, S_{V}$ at $N_{V}$ (fig. $\left.6 b\right)$.

\section{Conclusion.}

L'échantillonnage (orientation du plan de coupe-épaisseur et choix des coupes, etc.) demeure la difficulté majeure de l'analyse quantitative des tissus principalement lorsque les cellules sont réparties selon une direction préférentielle. On ne peut donc pas appliquer systématiquement et sans précaution les méthodes présentées dans cet article car elles supposent toutes la répartition au hasard des particules dans le tissu. On peut regretter que ces méthodes n'aient pas une base probabiliste plus rigoureuse et que les auteurs n'explicitent pas mieux le terme hasard qui prend ainsi différentes significations selon les utilisateurs.

En réalité, aucun procédé d'analyse quantitative ne peut donner le nombre absolu et réel de particules présentes dans un matériel biologique. Seule une méthode probabiliste d'application simple tenant compte de la forme, des dimensions des particules et de leur répartition spatiale peut procurer des estimations représentatives du tissu analysé et permettre également l'estimation d'un indice de précision. Les formules proposées fournissent donc une approximation du nombre et, malgré leurs imperfections, permettent néanmoins la comparaison fructueuse d'échantillons soumis à des traitements différents grâce à la possibilité de standardisation. Cependant, l'estimation du nombre de particules par unité de volume donne une information limitée puisque sa variation peut s'expliquer par l'augmentation des tailles ou par l'accroissement des espaces entre particules, le nombre total restant constant. Pour une analyse plus fine des phénomènes, il convient donc de compléter cette information par l'estimation du nombre total ainsi que par celles des dimensions des particules. Actuellement, les méthodes stéréologiques classiques sont les mieux adaptées pour l'analyse quantitative des tissus ou organes. 


\section{Références}

ABERCROMBIE M., 1946. Estimation of nuclear population from microtome sections. Anat. Rec., 94, 239-247.

AHERNE W., 1967. Methods of counting discrete tissue components in microscopical sections. J. Royal Micr. Soc., 87, 493-508.

DE HOFF R. T., 1968. Measurement of number and average size in volume, 128-147. In R. T. DE HOFF, F. N. RHINES. Quantitative microscopy. Mc Graw-Hill, Book Company, New York.

DE HOFF R. T., RHINES F. N., 1961. Determination of number of particles per unit volume from measurements made on random plane sections : the general cylinder and the ellipsoid. Trans. Amer. Metall. Soc. AIME, 221, 975-998.

DEWEY W. C., FUHR M. A., 1976. Quantification of mitochondria during the cell cycle of chinese hamster cells. Exp. Cell Res., 99, 23-30.

DORNFELD E. J., SLATER D. W., SHEFFE H., 1942. A method for accurate defermination of volume and cell numbers in small organs. Anat. Rec., 82, 255-259.

DUNNILL M. S., 1962. Postnatal growth of the lung. Thorax, 17, 329-333.

DUNNILL M. S., 1964. Evaluation of a simple method of sampling the lung for quantitative histological analysis. Thorax, 19, 443-448.

EBBESSON S., TANG D., 1965. A method for estimating the number of cells in histological sections. J. Roy. Micr. Soc., 84, 449-464.

ELIAS H., HENNIG A., SCHWARTZ D. E., 1971. Stereology : applications to biomedical research. Physiol. Rev., 51, 158-200.

FLODERUS S., 1944. Untersuchungen uber den Bau der menschlichen Hypophyse mit besonderer Beruchsichtigung der quantitativen mikromorphologischen Verhältnisse. Acta pathol. microbiol. scand., suppl. 53.

FULLMAN R. L., 1953. Measurement of particle sizes in opaque bodies. J. Metals, 5, 447-452.

FREEDMAN L. S., 1974. A note on Aherne's method of counting tissue components in relatively thick sections. J. Microsc., 100, 219-225.

HELANDER H., BLOOM G. D., 1974. Quantitative analysis of mast cell structure. J. Microsc., 100, 315321.

LINDBERG L. G., VORWERK P., 1970. On calculating volumes of transected bodies from two dimensional micrographs. Lab. Invest., 23, 315-317.

LOUD A. V., 1968. A quantitative stereological description of the ultrastructure of normal rat liver parenchymal cells. J. Cell Biol., 37, 27-46.

LOUD A. V., BARANY W. C., PACK B. A., 1965. Quantitative evaluation of cytoplasmic structures in electron micrographs. Lab. Invest., 14, 996-1008.

MARRABLE A. W., 1962. The counting of cells and nuclei in microtome sections. Quarf. J. Micr. Sci., 103, 331-341.

MATHIEU O., MESSIER P. E., 1973. Principes et méthodes de la stéréologie tels qu'appliqués au domaine de l'ultrastructure cellulaire. Rev. Can. Biol., 32, 31-63.

MAYHEW T. M., 1972. A comparison of several methods for stereological determination of the numbers of organelles per unit volume of cytoplasm. J. Microsc., 96, 37-44.

MAUSLE E., SCHERRER H., 1974. Ultramorphometric studies regarding the effect of sex-hormones on the adrenal cortex of the rat. Cell Tiss. Res., 149, 113-120.

NOURTIER C., 1971. Méthodes d'estimation quantitative au service de la cytologie. Ann. Biol., 10, 242-278. 
NUSSDORFER G. G., REBUFFAT P., MAZZOCHI G., BELLONI A. S., MENEGHELLI V., 1974. Investigations on adrenocortical mitochondria turnover. I. - Effects of chronic treatment with ACTH on the size and number of rat zona fasciculata mitochondria. Cell Tiss. Res., 150, 79-94.

SOLARI A., 1973. Etude quantitative d'organes ou de tissus. I. - Méthodes d'estimation des volumes. Ann. Biol. anim. Bioch. Biophys., 13, 247-265.

SOLARI A., 1975. Etude quantitative d'organes ou de tissus. II. - Méthodes de mesure des surfaces de structure cellulaire. Ann. Biol. anim. Bioch. Biophys., 15, 589-605.

WEIBEL E., 1963. Principles and methods for the morphometric study of the lung and other organs. Lab. Invest., 12, 131-155.

WEIBEL E., 1969. Morphometric cytology. Int. Rev. Cytol., 26, 235-302.

WEIBEL E., GOMEZ D. M., 1962. A principle for counting tissue structures on random sections. J. appl. Physiol., 17, 343-348.

WEIBEL E., KISTLER G. S., SCHERLE W. F., 1966. Practical stereological methods for morphometric cytology. J. Cell Biol., 30, 23-38. 